\title{
Green synthesis, size control, and antibacterial activity of silver nanoparticles on chitosan films
}

\author{
Cheng-Ho Chen ${ }^{1} \mathbb{D} \cdot$ Yin-Chen Lin ${ }^{1} \cdot$ Ching-Fong Mao ${ }^{1} \cdot$ Wei-Tung Liao ${ }^{1}$
}

Received: 16 January 2019 / Accepted: 28 April 2019 / Published online: 7 May 2019

(C) Springer Nature B.V. 2019

\begin{abstract}
Silver nanoparticles (AgNPs) synthesized on the surface of chitosan (CS) films using ultraviolet (UV) and natural light irradiation reduction methods were studied. Field-emission scanning electron microscopy revealed that the diameter of some AgNPs synthesized by the UV irradiation reduction method lay in the range of 5-15 nm. These AgNPs were isolated and dispersed on the surface of CS film. Meanwhile, some AgNPs aggregated to form larger cubic and irregular AgNPs with average size of 50-200 $\mathrm{nm}$. Compared with those synthesized via the UV irradiation reduction method, AgNPs synthesized by the natural light irradiation reduction method were found to be much more individually isolated and uniformly dispersed on the CS film surface. The AgNPs synthesized using the natural light irradiation reduction method had spherical shape with average size of 10-30 nm. Thus, the size and morphology of AgNPs on the CS films (CS-AgNPs films) can be controlled by choosing an appropriate irradiation reduction method. The results of antibacterial testing illustrated that the CS-AgNPs films synthesized by both the ultraviolet and natural light irradiation reduction methods exhibited strong antibacterial activity against Escherichia coli.
\end{abstract}

Keywords Chitosan $\cdot$ Silver nanoparticle $\cdot$ Irradiation reduction $\cdot$ Antibacterial activity

\section{Introduction}

Chitin, a linear polysaccharide composed of (1-4)-linked 2-acetamido-2-deoxy- $\beta$-Dglucopyranose units, is the second most prevalent form of polymerized carbon in Nature. Its derivative, chitosan (CS), is prepared by deacetylation and depolymerization of native chitin, (partial) deacetylation of chitin in the solid state under alkaline

Cheng-Ho Chen

chchen@stust.edu.tw

1 Department of Chemical and Materials Engineering, Southern Taiwan University of Science and Technology, No. 1, Nan-Tai Street, Yong-Kang District, Tainan City 710, Taiwan 
conditions (concentrated $\mathrm{NaOH}$ ), or enzymatic hydrolysis in the presence of a chitin deacetylase [1]. The amine group on the CS backbone is proportional to its degree of deacetylation (DD). CS displays unique polycationic, chelating, and film-forming properties due to the presence of active amine and hydroxyl functional groups. CS also exhibits excellent biodegradability, biocompatibility, and bioactivity. Due to these characteristics, CS is widely used in many fields, such as medicine, food, chemical industry, pharmaceuticals, nutrition, agriculture, etc. [2-6].

Nowadays, noble-metal nanoparticles have attracted considerable attention because of their possible applications in microelectronics [7]. In particular, silver nanoparticles (AgNPs) exhibit unique electronic, catalytic, optical, and other physical and chemical properties [8]. Therefore, AgNPs have been applied in many fields, including photonics, microelectronics, photocatalysis, lithography, and surfaceenhanced Raman spectroscopy [9]. Silver or silver ions, even at low concentrations, show powerful antibacterial activity and are thus commonly used in the medical field [10-13].

Synthesis of AgNPs has been widely studied, and their stability and dispersion are crucial. Recently, incorporation of AgNPs into polymers has been proposed. Immobilizing AgNPs on matrix polymers, also known as nanometal-polymer hybrids, efficiently prevents AgNPs aggregation. In addition, the biocompatibility of the resultant product can be improved [14-16]. Two major routes have been adopted for reducing silver ions to silver in industry. One approach is to use the chemical reduction method [17-20]; For instance, Qiao et al. reported that well-dispersed spherically shaped AgNPs with size of 20-80 nm can be fabricated by reducing silver nitrate with glucose in the presence of polyvinylpyrrolidone (PVP), a protective agent [17]. Yang et al. prepared various metal-CS nanocomposites in aqueous solutions. The metals studied were silver $(\mathrm{Ag})$, gold $(\mathrm{Au})$, platinum $(\mathrm{Pt})$, and palladium (Pd). The metal nanoparticles were formed by reduction of corresponding metal salts with $\mathrm{NaBH}_{4}$ in the presence of CS [20].

The other route for reducing silver ions to silver is by physical reduction methods [21-29]; For instance, Wei et al. [21] reported preparation of gold nanomaterials by thermal reduction methods in chitosan-acetic acid-water solution. Chen et al. [22] prepared poly[butyl acrylate-co-(glycidyl methacrylate-iminodiacetic acid)] microspheres by batch soap-free emulsion copolymerization of $n$-butyl acrylate with a chelating vinyl monomer. The flexible and water-insoluble films obtained from latex were used to adsorb silver ions from aqueous solution. Thus, AgNPs on the surface of the film were prepared through reduction of the copolymer-silver ion complexes by using UV light irradiation. Zhang et al. [23] manufactured a polyacrylonitrile/ silver nanoparticle composite by using the simultaneous polymerization reduction approach. They also used the UV light irradiation method for reducing silver ions to AgNPs. El-Naggar et al. [26] reported clean and high-throughput production of AgNPs mediated by soy protein via solid-state synthesis. They also reported solvent-free and one-pot synthesis of silver and zinc oxide nanoparticles [27]. Huang et al. [29] reported synthesis of AgNPs by a $\gamma$-ray irradiation reduction method with low-molecular-weight CS as stabilizer and coreductant.

Compared with the chemical reduction method, the physical irradiation method does not require chemical reducing agents and the process steps are simple. The 
physical irradiation method is a convenient and "green" approach to synthesize AgNPs. Therefore, two physical irradiation reduction methods, i.e., based on UV and natural light irradiation, were chosen in this study. Meanwhile, since CS exhibits excellent biodegradability and biocompatibility, it was chosen as a surface material to demonstrate a facile and low-cost way to synthesize AgNPs on the surface of CS films (CS-AgNPs films).

The morphology and composition of the AgNPs fabricated on the surface of CS films via the UV or natural light irradiation reduction method were observed by field-emission scanning electron microscopy (FE-SEM) and energy-dispersive X-ray spectroscopy (EDS). Moreover, the chemical structure of pure CS and CS-AgNPs films were examined and compared using attenuated total reflection Fourier-transform infrared (ATR-FTIR) spectrometry. Furthermore, the antibacterial properties of the CS-AgNPs films against $E$. coli were also examined.

\section{Experimental}

\section{Materials}

Chitosan with 95\% DD (prepared from $\beta$-chitin) was obtained from Ohka Enterprises Co., Ltd., Taiwan. The molecular weight of the CS was about 200,000 Da. Acetic acid was purchased from Union Chemical Works Ltd., Taiwan. Silver nitrate was purchased from Showa Chemical Industry Co., Ltd., Japan.

\section{Fabrication of CS film}

CS powder was added to $0.1 \mathrm{M}$ acetic acid solution and stirred to form $2.5 \mathrm{wt} \%$ clear CS solution. This clear solution was then poured into a polypropylene (PP) Petri dish. After settling for $48 \mathrm{~h}$, this CS solution was debubbled and placed in an oven at $60{ }^{\circ} \mathrm{C}$ to dry for $24 \mathrm{~h}$. The resulting material was then vacuum dried at $80{ }^{\circ} \mathrm{C}$ for $24 \mathrm{~h}$ to form a CS film.

\section{Reduction of silver ions on surface of CS film}

The CS film was cut to $3 \mathrm{~cm} \times 3 \mathrm{~cm}$, then immersed in $0.01 \mathrm{M}$ silver nitrate aqueous solution for $1 \mathrm{~h}$. After $1 \mathrm{~h}$, the surface of the CS film with silver ions was washed with deionized (DI) water several times to remove residual silver ions. After washing, the CS film with silver ions was dried in an oven at $60{ }^{\circ} \mathrm{C}$ for $24 \mathrm{~h}$. A $30-\mathrm{W}$ column-like mercury lamp $(\lambda=254 \mathrm{~nm})$ was used as an UV irradiation source. The UV and natural light irradiation reduction methods were then applied separately to reduce silver ions to silver nanoparticles (AgNPs). For the UV light irradiation reduction process, the dried CS film was placed under UV light $(254 \mathrm{~nm})$ about $20 \mathrm{~cm}$ away for $1 \mathrm{~h}$. For the natural light irradiation reduction process, it was placed in the laboratory under natural light for $72 \mathrm{~h}$. 


\section{ATR-FTIR analysis}

The functional groups of pure CS and CS-AgNPs films were characterized and analyzed using an ATR-FTIR spectrometer (Spectron One; PerkinElmer, USA) in the wavelength range of $400-4000 \mathrm{~cm}^{-1}$ at scanning frequency of $32 \mathrm{scans} / \mathrm{s}$.

\section{FE-SEM examination}

The morphology of CS-AgNPs films was examined using field-emission scanning electron microscopy (FE-SEM) (JSM 6700F; JEOL Ltd., Japan). Each sample was coated with a gold-palladium film. The presence of Ag was analyzed by energy-dispersive X-ray spectrometry (EDS).

\section{Antibacterial testing of CS-AgNPs film against E. coli}

Three flasks, each containing $90 \mathrm{ml}$ sterilized water, were prepared. E. coli solution $(10 \mathrm{ml})$ was added to each flask. CS-AgNPs films prepared by either the UV or natural light irradiation reduction method were introduced into one of the flasks, while pure CS film was added to another. The third flask was used as a blank control. The materials in each of these three flasks were stirred using an orbital shaker for $1 \mathrm{~h}$, then further diluted to $1 \times 10^{-5}$ of the initial concentration. After that, $1 \mathrm{ml}$ of each diluted solution was seeded into a Petri dish, and Luria-Bertani agar was added to each dish. The plates were then incubated at $37{ }^{\circ} \mathrm{C}$ for $12 \mathrm{~h}$. Bacterial colonies were counted to evaluated the surviving numbers of $E$. coli [30].

\section{Results and discussion}

\section{ATR-FTIR analyses of pure CS and CS-AgNPs films}

Figure 1 shows the ATR-FTIR spectra of the surfaces of the pure CS and the CSAgNPs films synthesized by the UV and natural light irradiation reduction methods. For pure CS film, the signals at about 1030 and $1060 \mathrm{~cm}^{-1}$ confirm the presence of $\mathrm{C}-\mathrm{O}$ bonds [31], while that at about $1550 \mathrm{~cm}^{-1}$ corresponds to bending vibration of $-\mathrm{NH}_{2}$ group, and that at about $1630 \mathrm{~cm}^{-1}$ to bending vibration of the amide group, while those at about $3200-3600 \mathrm{~cm}^{-1}$ correspond to the combination of $-\mathrm{OH}$ and $-\mathrm{NH}$ stretching vibrations. Since the number of amine groups on the backbone of CS is proportional to its DD, the chelating ability of CS is also proportional to the DD. After the reduction of silver ions chelated on the surface of the CS film in AgNPs by the UV or natural light irradiation reduction process, the observed ATR-FTIR spectrum of the CS-AgNPs film was significantly different from that of the pure CS film. The intensities of characteristic peaks of the bending vibration of $-\mathrm{NH}_{2}$ group, the bending vibration of amide group, and the 


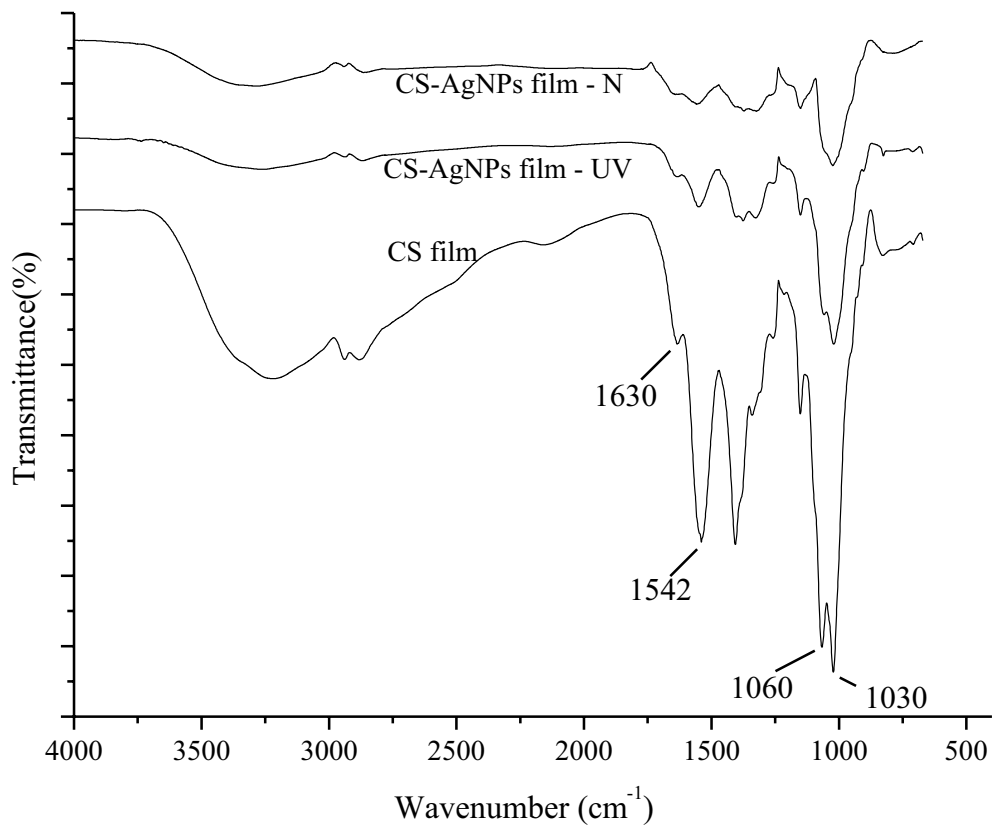

Fig. 1 FTIR spectra of CS and CS-AgNPs films (CS-AgNPs film-N: synthesized by natural light irradiation reduction method; CS-AgNPs film-UV: synthesized by UV light irradiation reduction method)

combination of $-\mathrm{OH}$ and $-\mathrm{NH}$ functional groups were all significantly smaller for the CS-AgNPs film compared with the pure CS film, indicating successfully synthesis of AgNPs on the surface of the CS film.

\section{FE-SEM and EDX results}

Figure 2 shows a FE-SEM micrograph of the CS-AgNPs film synthesized by the UV light irradiation reduction method. Many spherical, cubic, and irregular AgNPs were observed on the surface of the CS film. The enlarged panel in Fig. 2 clearly shows individually isolated spherical AgNPs with diameter in the range of 5-15 nm. However, some spherical AgNPs aggregated to form larger cubic and irregular AgNPs. The diameter of the cubic and irregular AgNPs lay in the range of 50-200 nm. This was because the irradiation energy of the UV light was strong enough to enhance the reduction rate of silver ions. The spontaneous formation of larger cubic and irregular AgNPs observed here resembles that observed in the ripening process of AgNPs prepared using chemical reduction methods. The ripening process is a transformation of AgNPs that occurs after AgNP formation by chemical reduction reactions [32]. The stability of atoms in an AgNP decreases concomitantly with decreasing particle size because of the increase in the surface energy. In the case of spherical AgNPs, because they consist of high-order unstable facets with high surface energies, the total surface area of such unstable facets increases concomitantly with decreasing 


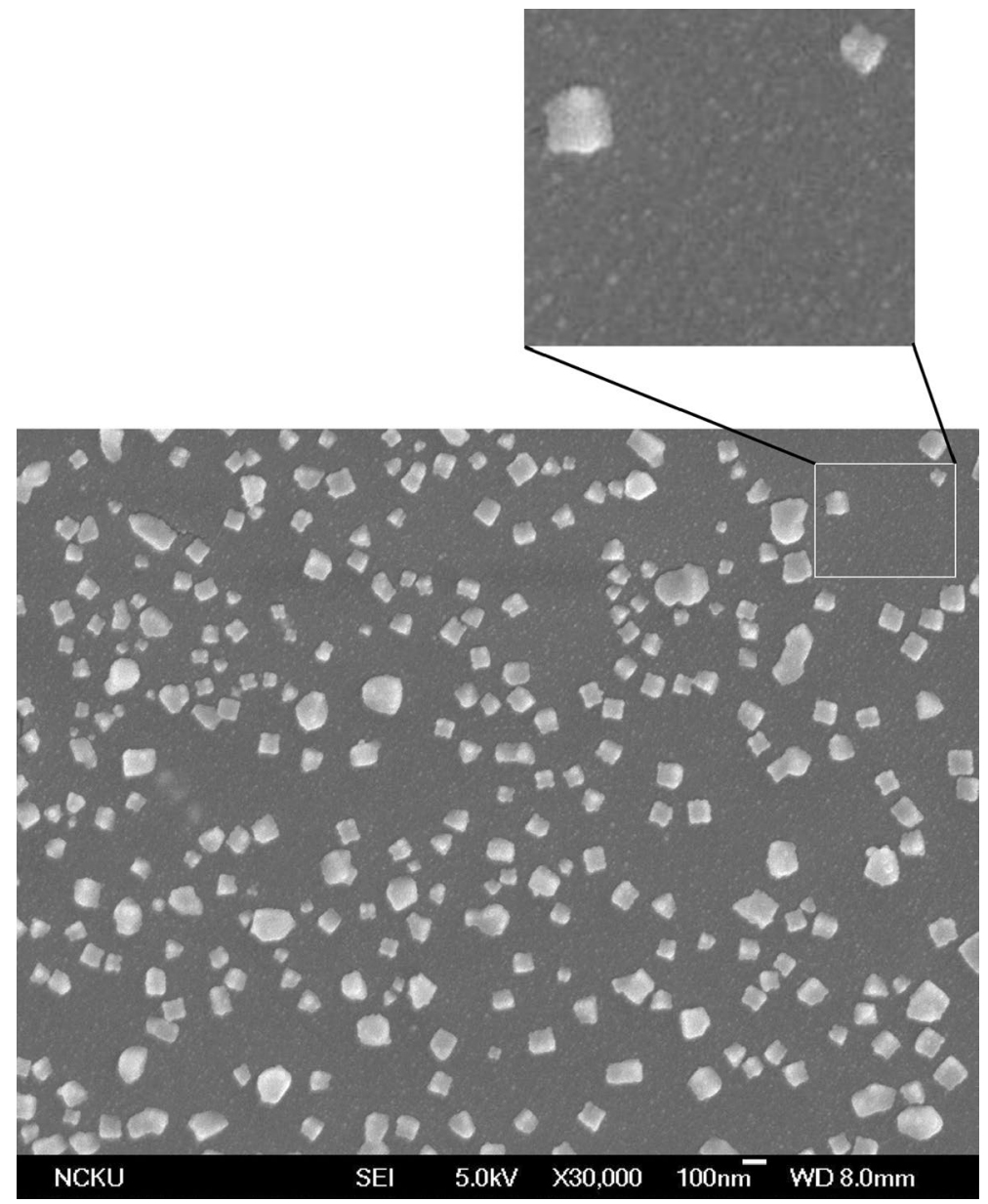

Fig. 2 FE-SEM image $(\times 30,000)$ of the surface of CS-AgNPs film synthesized by UV light irradiation reduction method

particle size. Thus, some spherical AgNPs tend to agglomerate into larger cubic and irregular AgNPs [33].

Figures 3 and 4 show FE-SEM micrographs of the CS-AgNPs film synthesized by the natural light irradiation reduction at magnification of $\times 30,000$ and $\times 50,000$, respectively. Many spherical AgNPs were observed on the surface of CS film. The individually isolated AgNPs had diameter in the range of 10-30 nm. Only a few AgNPs aggregated to form larger AgNPs, with diameter in the range of 50-200 nm.

Comparing the morphology of the AgNPs synthesized by the natural light irradiation reduction method (Fig. 3) with that of those synthesized by the UV light irradiation reduction method (Fig. 2) reveals that the AgNPs produced via the natural light irradiation reduction method were much more individually isolated and uniformly distributed on the surface of the CS film. Conversely, when irradiated 


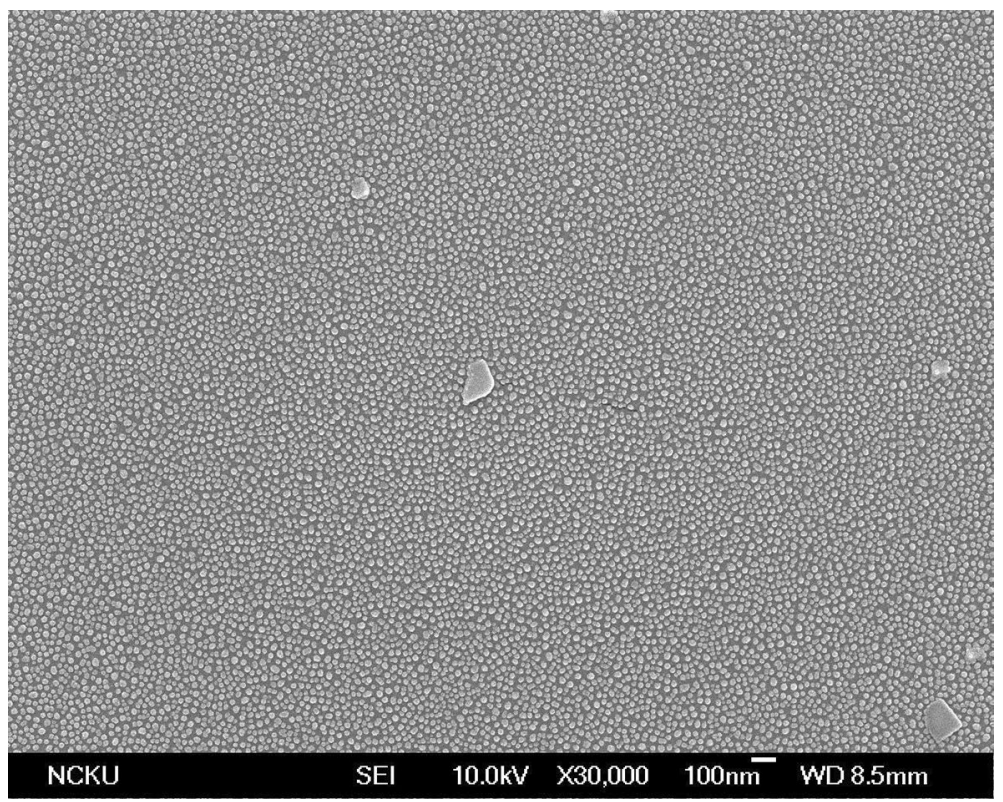

Fig. 3 FE-SEM image $(\times 30,000)$ of surface of CS-AgNPs film synthesized by natural light irradiation reduction method

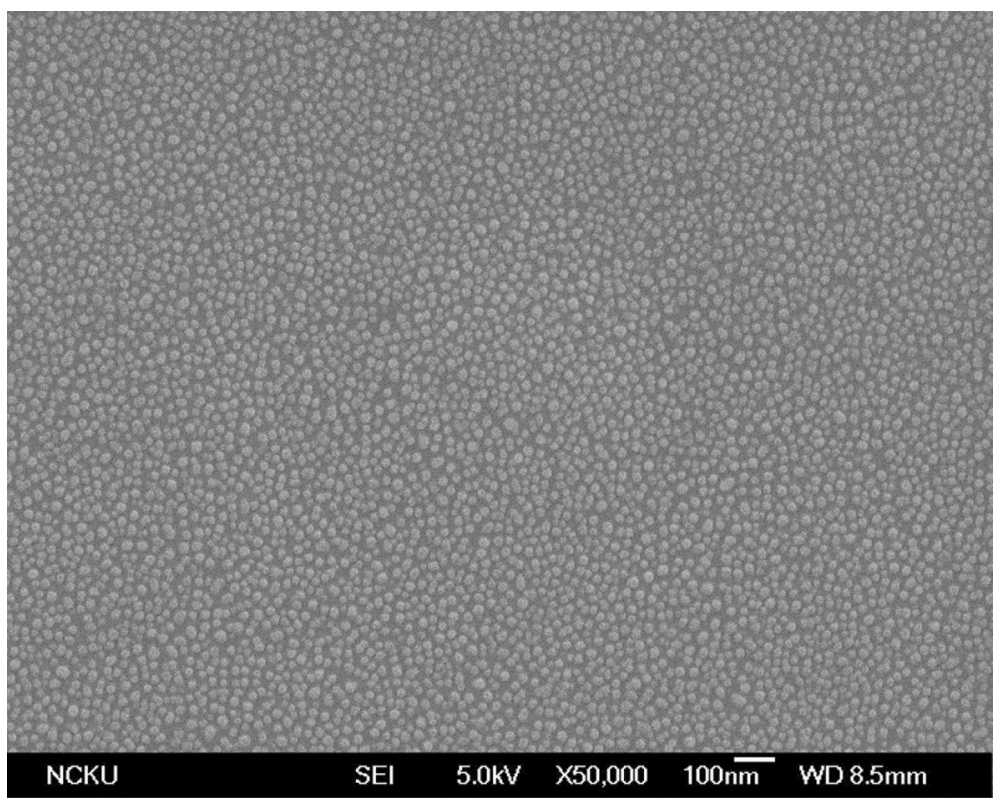

Fig. 4 FE-SEM image $(\times 50,000)$ of surface of CS-AgNPs film synthesized by natural light irradiation reduction method 
with UV light, the silver ions quickly absorb the stronger radiant energy, causing their reduction to form spherical AgNPs and also causing some spherical AgNPs to agglomerate into larger cubic and irregular AgNPs. This is because the irradiation energy of natural light is more moderate than that of UV light. Therefore, silver ions were slowly reduced to spherical AgNPs via the natural light irradiation reduction method and not easily aggregated to form larger cubic and irregular AgNPs.

Figure 5a shows the EDX results for the surface of the CS-AgNPs film prepared by the UV irradiation reduction method. The results demonstrate the presence of silver element on the CS film, confirming that the nanoparticles on the surface of the CS film were AgNPs. The EDX results for the CS-AgNPs film synthesized by the natural light irradiation reduction method (Fig. 5b) were similar to the results for the sample obtained by the UV light irradiation reduction method.

\section{Antibacterial results for pure CS and CS-AgNPs films against $E$. coli}

Figure 6 shows the antibacterial activity of the CS-AgNPs film against E. coli. Figure 6 a shows the blank control of the antibacterial test. The surviving number of bacteria was found to be about 80 after incubation. When the E. coli solution was
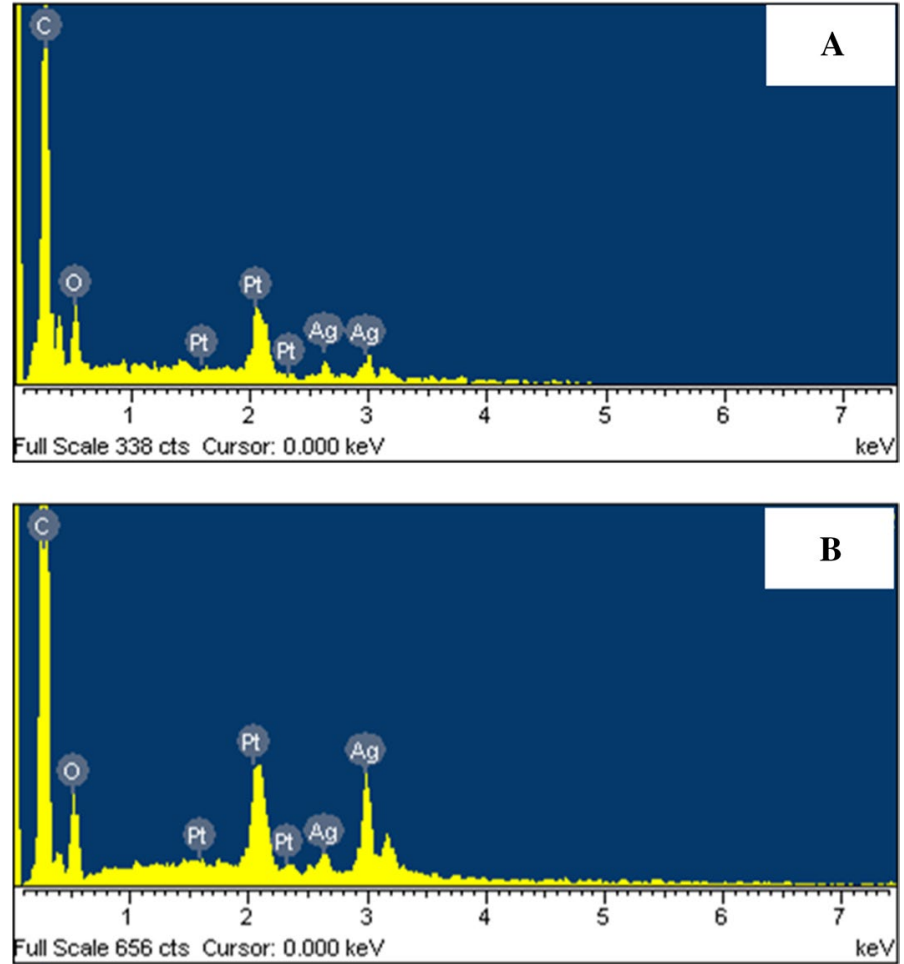

Fig. 5 EDX spectra of CS-AgNPs film synthesized by a UV light and $\mathbf{b}$ natural light irradiation reduction methods 


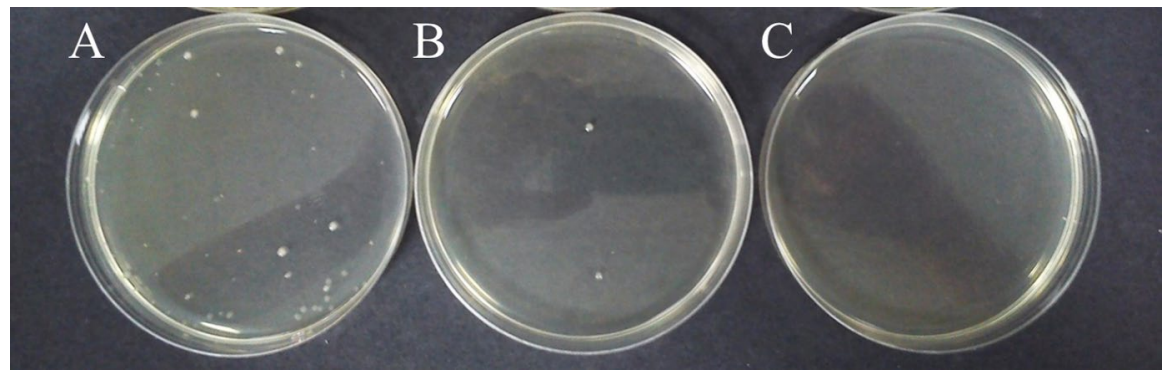

Fig. 6 Antibacterial ability of a blank control, b pure CS film, and c CS-AgNPs film

sterilized with pure CS film, the surviving number was reduced to about 5 (Fig. 6b). This result indicates that the pure CS film could effectively prevent growth of E. coli. One of the reasons for the antimicrobial character of chitosan is its positively charged amino group, which interacts with negatively charged microbial cell membranes, leading to leakage of proteinaceous and other intracellular constituents from the microorganism [34]. Furthermore, when the E. coli solution was sterilized with CS-AgNPs film, the average number of surviving bacterium was significantly decreased to less than 1 (Fig. 6c). This observation indicates that the CS-AgNPs film can kill almost $100 \%$ of E. coli. The sterilization ability of the CS-AgNPs film synthesized by the UV irradiation reduction and natural light irradiation reduction methods was the same. This is because both CS and AgNPs have good antibacterial effect. The CS-AgNPs film combines CS with AgNPs and therefore exhibits excellent antibacterial effect. Sondi and Salopek-Sondi reported that AgNPs with average size of $12 \mathrm{~nm}$ exhibited strong antimicrobial activity [35]. Son et al. [36] found that cellulose acetate nanofibers containing AgNPs with diameter of $21 \mathrm{~nm}$ could kill different kinds of bacteria efficiently. In this study, although the diameter of the small fraction of AgNPs was greater than $30 \mathrm{~nm}$, the CS-AgNPs film still showed strong antibacterial activity against $E$. coli.

\section{Conclusions}

A simple, convenient, and "green" approach to synthesize CS-AgNPs film via either the UV light or natural light irradiation reduction method was demonstrated. FE-SEM revealed that many AgNPs were formed on the surface of CS film by both irradiation reduction methods. Also, most of the AgNPs formed via the UV light irradiation reduction method were individually isolated and their diameter was in the range of 5-15 nm. Meanwhile, some AgNPs aggregated to form larger cubic and irregular AgNPs with diameter in the range of 50-200 $\mathrm{nm}$. On the other hand, it was observed that use of the natural light irradiation method reduced the probability of aggregate formation, thereby increasing the number of isolated and spherical AgNPs with diameter in the range of 10-30 nm. This indicates that the natural light irradiation reduction method synthesizes much more individually isolated and uniformly distributed AgNPs on the surface of the CS film. Thus, based on these observations, it is concluded that the size and 
morphology of AgNPs grown on CS film can be controlled by choosing an appropriate irradiation reduction method. The CS-AgNPs films synthesized by the UV light and natural light irradiation reduction methods showed strong antibacterial activity against E. coli, representing a promising candidate for use in a wide range of biomedical and general applications.

\section{References}

1. V. Zargar, M. Asghari, A. Dashti, ChemBioEng Rev. 2, 204 (2015)

2. D.W. Wei, W.Y. Sun, W.P. Qian, Y.Z. Ye, X.Y. Ma, Carbohydr. Res. 344, 2375 (2009)

3. J. Druet, A.E. Achari, J. Isaad, Res. Chem. Intermed. 41, 8855 (2015)

4. H.R. Lin, Y.S. Li, Y.J. Lin, Colloid Polym. Sci. 7, 1209 (2016)

5. E.D. Finashina, N.V. Kramareva, A.V. Kucherov, O.P. Tkachenko, L.M. Kustov, Res. Chem. Intermed. 42, 4907 (2016)

6. A.M. Abdelgawad, M.E. El-Naggar, S.M. Hudson, O.J. Rojas, Int. J. Biol. Macromol. 94(Part A), 96 (2017)

7. R.P. Andres, J.D. Bielefeld, J.I. Henderson, Science 273, 1690 (1996)

8. Y. Sun, Chem. Soc. Rev. 42, 2497 (2013)

9. K.G. Stamplecoskie, J.C. Scaiano, V.S. Tiwari, H. Anis, J. Phys. Chem. C 115, 1403 (2011)

10. H.Y. Kang, M.J. Jung, Y.K. Jeong, Korean J. Biotechnol. Bioeng. 15, 521 (2000)

11. A. Hebeish, M.H. El-Rafie, M.A. EL-Sheikh, A.A. Seleem, M.E. El-Naggar, Int. J. Biol. Macromol. 65, 509 (2014)

12. J.H. Yan, A.M. Abdelgawad, M.E. El-Naggar, O.J. Rojas, Carbohydr. Polym. 147, 500 (2016)

13. D. Medhat, J. Hussein, M.E. El-Naggar, M.F. Attia, M. Anwar, Y.A. Latif, H.F. Booles, S. Morsy, A.R. Farrag, W.K.B. Khalil, Z. El-Khayat, Biomed. Pharmacother. 91, 1006 (2017)

14. L.S. Wang, C.Y. Wang, C.H. Yang, C.L. Hsieh, S.Y. Chen, C.Y. Shen, J.J. Wang, K.S. Huang, Int. J. Nanomed. 10, 2685 (2015)

15. G.F. Prozorova, A.S. Pozdnyakov, N.P. Kuznetsova, Int. J. Nanomed. 9, 1883 (2014)

16. M. Rehan, M.E. El-Naggar, H.M. Mashaly, R. Wilken, Carbohydr. Polym. 182, 29 (2018)

17. H.S. Wang, X.L. Qiao, J.G. Chen, S.Y. Ding, Colloids Surf. A 256, 111 (2005)

18. K. Chou, C.Y. Ren, Mater. Chem. Phys. 64, 241 (2000)

19. S.A. Vorobyova, A.I. Lesnikovich, N.S. Sobal, Colloids Surf. A 152, 375 (1999)

20. H.Z. Huang, Q. Yuan, X.R. Yang, Colloids Surf. B Biointerfaces 39, 31 (2004)

21. D.W. Wei, W.P. Qian, Y. Shi, S.H. Ding, Y. Xia, Carbohydr. Res. 343, 512 (2008)

22. C.Y. Chen, C.Y. Chen, Thin Solid Films 484, 68 (2005)

23. Z. Zhang, L. Zhang, S. Wang, W. Chen, Y. Lei, Polymer 42, 8315 (2001)

24. X.M. Yang, Y. Lu, Mater. Lett. 59, 2484 (2005)

25. H.W. Lu, S.H. Liu, X.L. Wang, X.F. Qian, J. Yin, Z.K. Zhu, Mater. Chem. Phys. 81, 104 (2003)

26. M.E. El-Naggar, T.I. Shaheen, M.M.G. Fouda, A.A. Hebeish, Carbohydr. Polym. 136, 1128 (2016)

27. A.M. Abdelgawad, M.E. El-Naggar, W.H. Eisa, O.J. Rojas, J. Clean. Prod. 144, 501 (2017)

28. J. Hussein, M.E. El-Naggar, Y.A. Latif, D. Medhat, M. El Bana, E. Refaat, S. Morsy, Colloids Surf. B Biointerfaces 170, 76 (2018)

29. N.M. Huang, S.H. Radiman, N. Lim, P.S. Khiew, W.S. Chiu, K.H. Lee, A. Syahida, R. Hashim, C.H. Chia, Chem. Eng. J. 155, 499 (2009)

30. C.Y. Chen, C.L. Chiang, Mater. Lett. 62, 3607 (2008)

31. H. Zheng, Y. Du, J. Yu, R. Huang, L. Zhang, J. Appl. Polym. Sci. 80, 2558 (2001)

32. P.Y. Silvert, R.H. Urbina, K.T. Elhsissen, J. Mater. Chem. 7, 293 (1997)

33. T. Tsujia, M. Kikuchi, T. Kagawa, H. Adachi, M. Tsuji, Colloid Surf. A Physicochem. Eng. Asp. 529, 33 (2017)

34. F. Shahidi, J.K.V. Arachchi, Y.J. Jeon, Trends Food Sci. Technol. 10, 37 (1999)

35. I. Sondi, B. Salopek-Sondi, J. Colloid Interface Sci. 275, 177 (2004)

36. W.K. Son, J.H. Youk, W.H. Park, Carbohydr. Polym. 65, 430 (2006)

Publisher's Note Springer Nature remains neutral with regard to jurisdictional claims in published maps and institutional affiliations. 